\title{
PLANE POLOIDAL-TOROIDAL DECOMPOSITION OF DOUBLY PERIODIC VECTOR FIELDS. PART 1. FIELDS WITH DIVERGENCE
}

\author{
G. D. MCBAIN
}

(Received 22 October, 2003; revised 3 May, 2005)

\begin{abstract}
It is shown how to decompose a three-dimensional field periodic in two Cartesian coordinates into five parts, three of which are identically divergence-free and the other two orthogonal to all divergence-free fields. The three divergence-free parts coincide with the mean, poloidal and toroidal fields of Schmitt and Wahl; the present work, therefore, extends their decomposition from divergence-free fields to fields of arbitrary divergence. For the representation of known and unknown fields, each of the five subspaces is characterised by both a projection and a scalar representation. Use of Fourier components and wave coordinates reduces poloidal fields to the sum of two-dimensional poloidal fields, and toroidal fields to the sum of unidirectional toroidal fields.
\end{abstract}

\section{Introduction}

The conservation of mass constrains the velocity field $v(x, y, z)$ of an incompressible fluid to have zero divergence

$$
\nabla \cdot v \equiv \frac{\partial v_{x}}{\partial x}+\frac{\partial v_{y}}{\partial y}+\frac{\partial v_{z}}{\partial z}=0
$$

The analysis and solution of problems in incompressible fluid mechanics is often facilitated by using formulations in which this constraint is automatically satisfied. The best-known examples are the stream-functions for a plane

$$
v(x, y)=-\nabla \times\left\{\psi(x, y) \mathbf{i}_{z}\right\}
$$

\footnotetext{
'School of Aerospace, Mechanical and Mechatronic Engineering, The University of Sydney, Australia; e-mail: geordie.mcbain@aeromech.usyd.edu.au.

(C) Australian Mathematical Society 2005, Serial-fee code 1446-1811/05
} 
and axially symmetric flows [10, pages $63,125-126]$, (here, in general, $\mathbf{i}_{\varphi}$ is the $\varphi$-unit vector)

$$
\boldsymbol{v}(r, z)=-\nabla \times\left\{\frac{\psi(r, z)}{r} \mathbf{i}_{\theta}\right\} .
$$

Such reformulations are more difficult in three dimensions. For example, deriving the velocity field from a 'vector potential' [11]

$$
\boldsymbol{v}(x, y, z)=\boldsymbol{\nabla} \times \boldsymbol{\Psi}(x, y, z)
$$

eliminates the incompressibility constraint only by introducing another: that the vector potential itself is divergence-free. Note that the stream-functions in (1.1)-(1.2) are unique to within an additive scalar constant (independent of the specified twodimensional coordinates in each case) whereas any gradient can be added to the vector potential in (1.3) without changing the velocity. In electrodynamics the 'magnetic vector potential' of the divergence-free magnetic flux density also has a constraint on its divergence: the Lorentz condition [8, page 271].

A different approach is the poloidal-toroidal representation [3, page 622]:

$$
\boldsymbol{v}=(\nabla \times)^{2}\left(\Psi \mathbf{i}_{\rho}\right)+\nabla \times\left(\tau \mathbf{i}_{\rho}\right),
$$

where $\rho$ is the spherical radial coordinate. Encouraged by the success of this formulation in treating divergence-free flows in spherical cavities [13, 25], we are motivated here to examine the adaptation of the representation to flows in another important geometry in fluid mechanics: the infinite slot between parallel plane walls. Flows in this geometry include plane Couette and Poiseuille flow [4, 19, 21] and RayleighBénard $[2,20,24]$ and Waldmann $[14,28,22]$ convection. To avoid some of the analytical difficulties attending unbounded domains, theoretical and numerical treatments of such flows often employ periodic boundary conditions in the directions parallel to the wall $[19,23,24,26]$, and in the present work too attention is restricted to doubly periodic vector fields; namely (taking $x$ as normal, see Figure 1)

$$
v(x, y, z)=v\left(x, y+\frac{2 \pi}{k \cos \gamma}, z+\frac{2 \pi}{k \sin \gamma}\right)
$$

for all $y$ and $z$, and $x$ in some interval, say $\left(x_{0}, x_{1}\right)$. Doubly periodic (hereafter $d . p$.) fields are also useful in studies of heterogeneous media [15].

The analogue of (1.4) for the slot is the poloidal-toroidal representation

$$
v=(\nabla \times)^{2}\left(\Psi \mathbf{i}_{x}\right)+\nabla \times\left(\tau \mathbf{i}_{x}\right)
$$

used by Busse [2], Joseph [9, page 235], Moffatt [16, page 21] and Nagata [17]; however, as pointed out by Schmitt and Wahl [23], this is incomplete and a mean field must be added. This essential difference between the planar and spherical cases is a consequence of the 'hairy-sphere theorem' [18]: any continuous tangent vector field on a sphere must have a zero, and so, in particular, any constant field vanishes. 


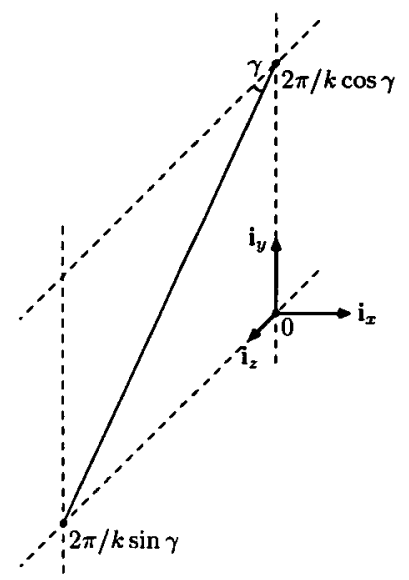

FIGURE 1. Standard Cartesian unit vectors, a period cell and periodicity angle $\gamma$.

1.1. Scalar and basis representations A scalar representation of a vector field $v$ is an expression of the form $v=\sum_{i} \mathbf{L}_{i} s_{i}$, where the $\mathbf{L}_{i}$ are linear operators independent of $v$ and the $s_{i}$ are scalar fields. A basis representation of a vector field is an expression of the form $v=\sum_{i} c_{i} \mathbf{a}_{i}$, where the $\mathbf{a}_{i}$ are vector fields independent of $v$ and the $c_{i}$ are scalar constants depending on $\boldsymbol{v}$. In particular, we are interested in representations for which divergence-free fields are easily identifiable; for example, because some fixed subset of the scalars $s_{i}$ or coefficients $c_{i}$ vanish.

A familiar and fundamental scalar representation of a vector field is

$$
v=\mathbf{i}_{x} v_{x}+\mathbf{i}_{y} v_{y}+\mathbf{i}_{z} v_{z}
$$

however, this may be inconvenient if the divergence is known to vanish.

1.2. Outline of the paper The plan of the present work is to derive consistent definitions for the poloidal and toroidal parts of d.p. fields, show that they form orthogonal subspaces, and then add more subspaces to give a complete orthogonal decomposition. For each subspace, the projection and a scalar representation are derived. General basis representations are not derived, since a good choice here depends on the nature of the problem under consideration and any approximation scheme that might be employed; however, orthonormal bases are presented in the example of Section 5.1 for the subspaces of the poloidal and toroidal subspaces that vanish on the slot walls (corresponding to zero velocity boundary conditions).

The representation derived here, when restricted to divergence-free fields, coincides with the poloidal-toroidal-mean field representation of Schmitt and Wahl [23]. The present representation is not restricted to divergence-free fields, but in it the projection of a vector field onto the divergence-free subspace is as trivial as projecting a point 
onto the $x y$-plane in Cartesian geometry.

The derivation is inspired by the work of Backus [1] on spherical poloidal and toroidal vector fields, in that we begin with a Helmholtz decomposition in two dimensions (there a spherical surface; here, in Section 2, a yz-period cell) and add a third normal dimension. The present derivation is simplified, however, by the use of suitably defined inner products and projections as in Neumann's [27] development of the geometry of Hilbert space.

\section{Doubly periodic plane fields}

Though we are primarily interested in three-dimensional vector fields, following Backus's [1] procedure for spherical poloidal-toroidal decomposition we begin by examining d.p. fields independent of $x$.

\subsection{Preliminaries}

2.1.1. Period cell averages, inner products and norms To discuss the geometry of the space of d.p. vector fields, we require an inner product [27, page 46]. This allows us to define orthogonality and so to partition the space in a natural and unambiguous way. Define

$$
\langle r, s\rangle_{\square} \equiv \frac{k^{2} \sin \gamma \cos \gamma}{4 \pi^{2}} \int_{0}^{2 \pi / k \sin \gamma} \int_{0}^{2 \pi / k \cos \gamma} r^{*} s \mathrm{~d} y \mathrm{~d} z,
$$

where $*$ denotes the complex conjugate. Then the plane average of a scalar $s$ over a period cell can be written as

$$
\langle s\rangle_{\square} \equiv\langle 1, s\rangle_{\square}
$$

For vectors, $\langle\boldsymbol{u}, \boldsymbol{v}\rangle_{\square} \equiv\langle\boldsymbol{u} \cdot \boldsymbol{v}\rangle_{\square} \equiv\left\langle u_{x}, v_{x}\right\rangle_{\square}+\left\langle u_{y}, v_{y}\right\rangle_{\square}+\left\langle u_{z}, v_{z}\right\rangle_{\square}$.

2.1.2. Projections Corresponding to each subspace of an inner product space is its orthogonal complement and its projection. Orthogonal sets of projections will be our principal tool in decomposing vector fields. An operator $\mathrm{P}$ is a projector if it is idempotent $\left(\mathrm{P}^{2} f=\mathrm{P} f\right)$ and Hermitian [27, page 77]

$$
\langle f, \mathrm{P} g\rangle=\langle\mathrm{P} f, g\rangle
$$

(or equivalently $\langle\mathrm{f}, \mathrm{P} g\rangle=\langle\mathrm{P} f, \mathrm{P} g\rangle$ ) for all $f$ and $g$ in the space.

Two projections are orthogonal if $\mathrm{PQ} f=\mathrm{QPf}=0$ for all $f$. The identity operator 1 is a projector, and so is the complement $(1-\mathrm{P})$ of any projector. Every projection is orthogonal to its complement.

Note that the scalar period cell average (2.1) is idempotent and Hermitian, and so is a projection on the space of plane d.p. scalar fields. 
2.1.3. Plane differential operators Backus [1] based his exposition of spherical poloidal and toroidal vector fields on certain differential operators defined on spherical surfaces. Here we introduce their plane analogues: the plane gradient of a scalar field and the plane divergence of a vector field

$$
\nabla_{\square} s \equiv \frac{\partial s}{\partial y} \mathbf{i}_{y}+\frac{\partial s}{\partial z} \mathbf{i}_{z} \quad \text { and } \quad \nabla_{\square} \cdot \boldsymbol{v} \equiv \frac{\partial v_{y}}{\partial y}+\frac{\partial v_{z}}{\partial z}
$$

and the plane curls of scalars and vectors

$$
\boldsymbol{\Lambda} s \equiv-\frac{\partial s}{\partial z} \mathbf{i}_{y}+\frac{\partial s}{\partial y} \mathbf{i}_{z} \quad \text { and } \quad \boldsymbol{\Lambda} \cdot \boldsymbol{v} \equiv \frac{\partial v_{z}}{\partial y}-\frac{\partial v_{y}}{\partial z} .
$$

In hydrodynamics, $\Lambda \psi$ gives a velocity field in the $y z$-plane from its stream-function [10, page 63] and $\Lambda \cdot v$ gives the normal $\left(i_{x}\right)$ component of vorticity [10, page 31] of the velocity field $\boldsymbol{v}$.

The plane Laplacian of a scalar field is

$$
\nabla_{\square}^{2} s \equiv \frac{\partial^{2} s}{\partial y^{2}}+\frac{\partial^{2} s}{\partial z^{2}}=\nabla_{\square} \cdot \nabla_{\square} s=\Lambda \cdot \Lambda s
$$

but the other combinations vanish:

$$
\nabla_{\square} \cdot \Lambda s=\Lambda \cdot \nabla_{\square} s=0 .
$$

Some other useful identities for d.p. scalar and vector fields are:

$$
\begin{aligned}
\left\langle s, \nabla_{\square} \cdot v\right\rangle_{\square} & =-\left\langle\nabla_{\square} s, \boldsymbol{v}\right\rangle_{\square}, \\
\langle s, \boldsymbol{\Lambda} \cdot \boldsymbol{v}\rangle_{\square} & =-\langle\boldsymbol{\Lambda} s, v\rangle_{\square}, \\
\left\langle s, \nabla_{\square}^{2} t\right\rangle_{\square}=-\left\langle\nabla_{\square} s, \nabla_{\square} t\right\rangle_{\square} & =-\langle\boldsymbol{\Lambda} s, \boldsymbol{\Lambda} t\rangle_{\square}=\left\langle\nabla_{\square}^{2} s, t\right\rangle_{\square} \quad \text { and } \\
\left\langle\nabla_{\square} s, \boldsymbol{\Lambda} t\right\rangle_{\square} & =0 .
\end{aligned}
$$

Equation (2.7) is analogous to (46b) of Backus [1] for spheres; it is essential for the plane Helmholtz decomposition (Section 2.2, below).

2.1.4. Trigonometric basis for scalar fields The scalar fields

$$
e_{\ell} \equiv \exp \left\{\sqrt{-1} k\left(l_{y} y \cos \gamma+l_{z} z \sin \gamma\right)\right\}
$$

(where the subscript $\ell$ stands for a pair of integers $l_{y}$ and $l_{z}$ ) form an orthonormal basis for the inner product space of d.p. scalar fields defined in a plane of constant $x$, that is,

$$
\left\langle e_{m}, e_{\ell}\right\rangle_{\square}= \begin{cases}1, & \ell=m ; \\ 0, & \text { otherwise }\end{cases}
$$




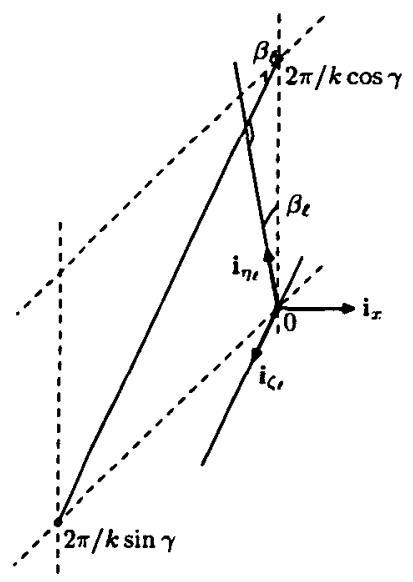

(a) $l_{z}=l_{y}$.

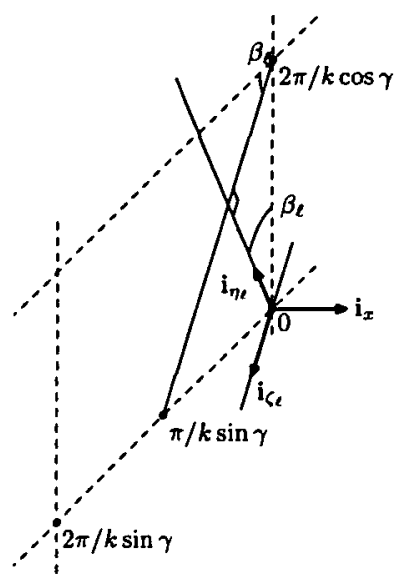

(b) $l_{z}=2 l_{y}$.

FIGURE 2. Wave Cartesian coordinates ( $k$ and $\gamma$ as in Figure 1.) For $l_{z}=0$, see Figure 1 , since then $\eta_{\ell}=y$ and $\zeta_{\ell}=z$.

A field $s$ with $\|s\|_{\square} \equiv\langle s, s\rangle_{\square}^{1 / 2}<\infty$ can be expanded in a Fourier series

$$
s(y, z)=\sum_{\ell}\left\langle e_{\ell}, s\right\rangle_{\square} e_{\ell} \equiv \sum_{l_{y}=-\infty}^{\infty} \sum_{l_{z}=-\infty}^{\infty}\left\langle e_{\ell}, s\right\rangle_{\square} e_{\ell}
$$

which converges in the norm. Here and elsewhere $\sum_{\ell}$ will be written to denote a summation over all integer values of $l_{y}$ and $l_{z}$.

2.1.5. Wave coordinates Manipulation of the trigonometric basis functions is facilitated by the introduction of the Cartesian 'wave coordinates'

$$
\left\{\begin{array}{l}
\eta_{\ell} \\
\zeta_{\ell}
\end{array}\right\}=\left[\begin{array}{cc}
\cos \beta_{\ell} & \sin \beta_{\ell} \\
-\sin \beta_{\ell} & \cos \beta_{\ell}
\end{array}\right]\left\{\begin{array}{l}
y \\
z
\end{array}\right\}
$$

where the wave angle $\beta_{\ell}$ is defined by $\kappa_{\ell} \cos \beta_{\ell}=k l_{y} \cos \gamma, \kappa_{\ell} \sin \beta_{\ell}=k l_{z} \sin \gamma$ and $\kappa_{\ell}=k\left(l_{y}^{2} \cos ^{2} \gamma+l_{z}^{2} \sin ^{2} \gamma\right)^{1 / 2}$. Their unit vectors

$$
\begin{aligned}
& \mathbf{i}_{\eta_{\ell}} \equiv \nabla_{a} \eta_{\ell}=\cos \beta_{\ell} \mathbf{i}_{y}+\sin \beta_{\ell} \mathbf{i}_{z} \text { and } \\
& \mathbf{i}_{\zeta_{\ell}} \equiv \nabla_{\square} \zeta_{\ell}=\Lambda \eta_{\ell}=-\sin \beta_{\ell} \mathbf{i}_{y}+\cos \beta_{\ell} \mathbf{i}_{z}
\end{aligned}
$$

are the standard Cartesian unit vectors rotated through $\beta_{\ell}$ (see Figure 2). This transformation is essentially that used by Squire [26] in his reduction to two dimensions of the equations governing infinitesimal three-dimensional disturbances to parallel shear flow. 
The trigonometric basis functions (2.8) become

$$
e_{\ell}=\exp \left(\sqrt{-1} \kappa_{\ell} \eta_{\ell}\right)
$$

and their derivatives are

$$
\begin{aligned}
\nabla_{\square} e_{\ell} & =\sqrt{-1} \kappa_{\ell} e_{\ell} \mathbf{i}_{\eta \ell}, \\
\Lambda e_{\ell} & =\sqrt{-1} \kappa_{\ell} e_{\ell} \mathbf{i}_{\zeta \ell} \quad \text { and } \\
\nabla_{\square}^{2} e_{\ell} & =-\kappa_{\ell}^{2} e_{\ell} .
\end{aligned}
$$

2.1.6. Poisson equation in the plane To deal with the orthogonal projections partitioning the space of plane d.p. vector fields in Section 2.2, we require some results [23] on the Poisson equation

$$
\nabla_{\square}^{2} u=-f
$$

where both $u$ and $f$ are d.p. Taking the inner product of both sides of (2.13) with a trigonometric basis function $e_{\ell}(2.10)$ gives $\kappa_{\ell}^{2}\left\langle e_{\ell}, u\right\rangle_{\square}=\left\langle e_{\ell}, f\right\rangle_{\square}$. Thus, provided

$$
\left\langle e_{0}, f\right\rangle_{\square} \equiv\langle f\rangle_{\square}=0,
$$

a d.p. solution of (2.13) is

$$
u=-\nabla_{\square}^{-2} f \equiv \sum_{\ell}^{\prime} \kappa_{\ell}^{-2}\left\langle e_{\ell}, f\right\rangle_{\square} e_{\ell},
$$

a prime being inserted $\left(\sum_{\ell}^{\prime}\right)$ when the term for which $l_{y}=l_{z}=0$ is to be omitted from the summation. While any function $u+$ const. is also a d.p. solution of (2.13), (2.15) is the only one with zero mean over the period cell. This follows from (2.6) with $s=t$ equal to the difference of two zero-mean solutions. In general, $\nabla_{\square}^{-2}$ is only a pseudoinverse; however, it is a true inverse if restricted to the subspace defined by (2.14), which will suffice here.

2.2. Helmholtz decomposition in the plane We are now ready to show that the space of plane doubly periodic vector fields (that is, those for which $v_{x}=0$ ) can be decomposed into three orthogonal subspaces: mean fields $\mathscr{M}_{\square}$, plane gradients $\mathscr{Q}_{0}$ and plane curls $\mathscr{T}_{0}$. This is analogous to the Helmholtz decomposition in space [10, page 208]. Each subspace is defined by a projector. Two of the projectors are defined as differential operators, so for nondifferentiable fields they should be understood as applying termwise to the Fourier expansion (2.9) via (2.11) or (2.12). Specifically,

$$
\begin{aligned}
& \mathrm{P}_{\mathscr{M}_{\square}} \boldsymbol{v}=\left\langle v_{y}\right\rangle_{\square} \mathbf{i}_{y}+\left\langle v_{z}\right\rangle_{\square} \mathbf{i}_{z}, \\
& \mathrm{P}_{\mathscr{Q}_{\square}} v=\nabla_{\square} \nabla_{\square}^{-2} \nabla_{\square} \cdot \boldsymbol{v} \text { and } \\
& \mathrm{P}_{\mathscr{T}_{\square}} \boldsymbol{v}=\boldsymbol{\Lambda} \nabla_{\square}^{-2} \boldsymbol{\Lambda} \cdot \boldsymbol{v} .
\end{aligned}
$$


The pseudoinverse Laplacians are well defined (2.14), by (2.4)-(2.5).

That each $\mathrm{P}$ is idempotent and Hermitian and that they are mutually orthogonal are straightforward consequences of the definitions and rules of Sections 2.1.1-2.1.3, particularly (2.2)-(2.7). Completeness follows from consideration of the expansion

$$
v=\left(\mathrm{P}_{\mathscr{M}_{\square}}+\sum_{\ell}^{\prime} \mathrm{P}_{\ell_{\square}}\right) v,
$$

where (for $l_{y}$ and $l_{z}$ not both zero) $\mathrm{P}_{\ell_{\square}} v \equiv\left\langle e_{\ell}, v_{\eta_{\ell}}\right\rangle_{\square} e_{\ell} i_{\eta_{\ell}}+\left\langle e_{\ell}, v_{\zeta_{\ell}}\right\rangle_{\square} e_{\ell} i_{\zeta_{\ell}}$ and the results

$$
\begin{aligned}
& \mathbf{P}_{\mathscr{M}_{\square}} \mathrm{P}_{\ell_{\square}} v=\mathrm{P}_{\ell_{\square}} \mathrm{P}_{\mathscr{M}_{\square}} v=0, \\
& \mathrm{P}_{\mathscr{Q}_{\square}} \mathrm{P}_{\ell_{\square}} v=\mathrm{P}_{\ell_{\square}} \mathrm{P}_{\mathscr{Q}_{\square}} v=\left\langle e_{\ell}, v_{\eta}\right\rangle_{\square} e_{\ell} \mathbf{i}_{\eta_{\ell}} \quad \text { and } \\
& \mathrm{P}_{\mathscr{T}_{\square}} \mathrm{P}_{\ell_{\square}} v=\mathrm{P}_{\ell_{\square}} \mathrm{P}_{\mathscr{T}_{\square}} v=\left\langle e_{\ell}, v_{\zeta}\right\rangle_{\square} e_{\ell} \mathbf{i}_{\boldsymbol{l}_{\ell}}
\end{aligned}
$$

which imply $\left(\mathrm{P}_{\mathscr{M}_{\square}}+\mathrm{P}_{\mathscr{Q}_{\square}}+\mathrm{P}_{\mathscr{T}_{\square}}\right) \mathbf{P}_{\ell_{\square}}=\mathrm{P}_{\ell_{\square}}\left(\mathrm{P}_{\mathscr{M}_{\square}}+\mathrm{P}_{\mathscr{Q}_{\square}}+\mathrm{P}_{\mathscr{T}_{\square}}\right)=\mathrm{P}_{\ell_{\square}}$.

\section{Doubly periodic space fields}

\subsection{Preliminaries}

3.1.1. Space inner products and averages The inner products and averages of Section 2.1.1 can be extended to three-dimensional yz-periodic fields [19, Eq. 12]:

$$
\begin{aligned}
\langle r, s\rangle & \equiv\left(x_{1}-x_{0}\right)^{-1} \int_{x_{0}}^{x_{1}}\langle r, s\rangle_{\square} \mathrm{d} x, \\
\langle s\rangle & \equiv\langle 1, s\rangle \quad \text { and } \\
\langle\boldsymbol{u}, \boldsymbol{v}\rangle & =\langle\boldsymbol{u} \cdot \boldsymbol{v}\rangle=\left\langle u_{x}, v_{x}\right\rangle+\left\langle u_{y}, v_{y}\right\rangle+\left\langle u_{z}, v_{z}\right\rangle .
\end{aligned}
$$

3.1.2. Space Poisson equation As in the planar case, some of the projections partitioning the space of space d.p. vector fields require the solution of a Poisson equation. For d.p. $u, f, b_{0}$ and $b_{1}$, consider

$$
\begin{aligned}
\nabla^{2} u & =-f \quad \text { and } \\
\left.u\right|_{x=x_{i}} & =b_{i}(y, z), \quad(i=0,1) \\
\text { or }\left.\quad \mathrm{D} u\right|_{x=x_{i}} & =b_{i}(y, z), \quad(i=0,1)
\end{aligned}
$$

(where $D \equiv \partial / \partial x$ ) and where

$$
\langle u\rangle_{\square}=\langle f\rangle_{\square}=\left\langle b_{0}\right\rangle_{\square}=\left\langle b_{1}\right\rangle_{\square}=0 .
$$


TABLE 1. Tripartite decomposition of d.p. vector fields.

\begin{tabular}{cll} 
Space & Projection & Scalar representation \\
\hline $\mathscr{M}$ & $\mathrm{P}_{\mathscr{M}} v \equiv\left\langle v_{x}\right\rangle_{\square} \mathbf{i}_{x}+\mathrm{P}_{\mathscr{M}_{\square}} v$ & $\boldsymbol{m}=U \mathbf{i}_{x}+V \mathbf{i}_{y}+W \mathbf{i}_{z}$, where \\
& & $\nabla_{\square} U=\nabla_{\square} V=\nabla_{\square} W=0$ \\
\hline $\mathscr{Q}$ & $\mathrm{P}_{\mathscr{Q}} v \equiv\left(v_{x}-\left\langle v_{x}\right\rangle_{\square}\right) \mathbf{i}_{x}+\mathrm{P}_{\mathscr{Q}_{\square}} v$ & $\begin{array}{l}\boldsymbol{q}=-r \mathbf{i}_{x}-\nabla_{\square} s, \text { where } \\
\langle r\rangle_{\square}=\langle s\rangle_{\square}=0\end{array}$ \\
& & $\begin{array}{l}\boldsymbol{t}=-\boldsymbol{\Lambda} \tau \equiv \nabla \times\left(\tau \mathbf{i}_{x}\right), \text { where } \\
\langle\tau\rangle_{\square}=0\end{array}$ \\
\hline $\mathscr{T}$ & $\mathrm{P}_{\mathscr{T}} v \equiv \mathrm{P}_{\mathscr{T}_{\square}} v$ &
\end{tabular}

Either Dirichlet (3.2) or Neumann (3.3) end conditions with the zero plane means (3.4) renders the solution of the space Poisson equation (3.1) unique, as follows from Green's identity

$$
\left\langle s, \nabla^{2} t\right\rangle-\frac{\left[\langle s, \mathrm{D} t\rangle_{\square}\right]_{x_{0}}^{x_{1}}}{x_{1}-x_{0}}=-\langle\nabla s, \nabla t\rangle=\left\langle\nabla^{2} s, t\right\rangle-\frac{\left[\langle\mathrm{D} s, t\rangle_{\square}\right]_{x_{0}}^{x_{1}}}{x_{1}-x_{0}}
$$

with $s=t$ equal to the difference of two solutions.

The Fourier components for $\ell \neq \mathbf{0}$ (the plane mean component vanishes) of the solution of (3.1) are

$$
\begin{aligned}
\tilde{u}_{\ell}(\xi)= & \left\{C_{\ell}\left\langle H(\xi-x) v_{\ell}(x), \tilde{f}_{\ell}\right\rangle+\tilde{b}_{0 \ell}\right\} w_{\ell}(\xi) \\
& +\left\{C_{\ell}\left\langle H(x-\xi) w_{\ell}(x), \tilde{f}_{\ell}\right\rangle+\tilde{b}_{1 \ell}\right\} v_{\ell}(\xi),
\end{aligned}
$$

where $\tilde{f}_{\ell} \equiv\left\langle e_{\ell}, f\right\rangle_{\square}($ etc. $)$ and $H$ is Heaviside's step function.

For Dirichlet end conditions (3.2)

$$
\begin{aligned}
C_{\ell} & =\frac{\sinh \kappa_{\ell}\left(x_{1}-x_{0}\right)}{\kappa_{\ell}}, \\
v_{\ell}(x) & =\frac{\sinh \kappa_{\ell}\left(x-x_{0}\right)}{\sinh \kappa_{\ell}\left(x_{1}-x_{0}\right)} \quad \text { and } \quad w_{\ell}(x)=\frac{\sinh \kappa_{\ell}\left(x_{1}-x\right)}{\sinh \kappa_{\ell}\left(x_{1}-x_{0}\right)},
\end{aligned}
$$

while for Neumann end conditions (3.3)

$$
\begin{aligned}
C_{\ell} & =-\kappa_{\ell} \sinh \kappa_{\ell}\left(x_{1}-x_{0}\right), \\
v(x) & =\frac{+\cosh \kappa_{\ell}\left(x-x_{0}\right)}{\kappa_{\ell} \sinh \kappa_{\ell}\left(x_{1}-x_{0}\right)} \quad \text { and } \quad w(x)=\frac{-\cosh \kappa_{\ell}\left(x_{1}-x\right)}{\kappa_{\ell} \sinh \kappa_{\ell}\left(x_{1}-x_{0}\right)} .
\end{aligned}
$$

3.2. A tripartite decomposition of d.p. vector fields The Helmholtz decomposition of d.p. plane vector fields (Section 2.2) can be extended to vector fields with nonzero normal $(x)$ components as in Table 1 . We call the three subspaces mean $(\mathscr{M})$, quasipoloidal $(\mathscr{Q})$ and toroidal $(\mathscr{T})$. The completeness and orthogonality of this tripartite 
decomposition follows from that of the plane Helmholtz decomposition and the simple treatment of the normal components along with the planarity of the operators involved. Table 1 also gives a scalar representation for the general member of each subspace, along with the conditions under which the scalars are unique. In each case, the definitions by the projection and scalar representation are equivalent.

3.2.1. Divergence in the tripartite decomposition If $\nabla \cdot v=0$, then $\nabla_{\square} \cdot v=-D v_{x}$ and $\mathrm{D}\left\langle v_{x}\right\rangle_{\square}=0$ by (2.4) and the quasipoloidal projection reduces to

$$
\begin{aligned}
\mathbf{P}_{\mathscr{Q}} \boldsymbol{v} & =\left(v_{x}-\left\langle v_{x}\right\rangle_{\square}\right) \mathbf{i}_{x}+\nabla_{\square} \nabla_{\square}^{-2} \mathrm{D} v_{x} \\
& =-\nabla_{\square}^{2} \boldsymbol{i}_{x}+\nabla_{\square} \mathrm{D} \Psi \equiv(\nabla \times)^{2}\left(\Psi_{\mathbf{i}_{x}}\right),
\end{aligned}
$$

say, with $\nabla_{\square}^{2} \Psi \equiv\left\langle v_{x}\right)_{\square}-v_{x}$, which is the same as Equation (7) of Schmitt and Wahl [23] (for divergence-free d.p. fields). They applied a tripartite decomposition equivalent to $\mathrm{P}_{\mathscr{K}}+\mathrm{P}_{\mathscr{Q}}+\mathrm{P}_{\mathscr{T}}$ to divergence-free fields, calling the three parts mean, poloidal and toroidal. The divergences of these projections of an arbitrary d.p. field are

$$
\begin{aligned}
\nabla \cdot \mathbf{P}_{\mathscr{M}} \boldsymbol{v} & =\mathrm{D}\left\langle v_{x}\right\rangle_{\square}=\langle\nabla \cdot \boldsymbol{v}\rangle_{\square}, \\
\nabla \cdot \mathbf{P}_{\mathscr{Q}} \boldsymbol{v} & =\mathrm{D}\left(v_{x}-\left\langle v_{x}\right\rangle_{\square}\right)+\nabla_{\square} \cdot \boldsymbol{v}=\nabla \cdot \boldsymbol{v}-\langle\nabla \cdot \boldsymbol{v}\rangle_{\square} \quad \text { and } \\
\nabla \cdot \mathbf{P}_{\mathscr{T}} \boldsymbol{v} & =\mathbf{0} .
\end{aligned}
$$

Thus, if restricted to divergence-free fields, the tripartite decomposition produces only divergence-free projections.

\section{Quinquepartite decomposition}

The Schmitt-Wahl decomposition is adequate for divergence-free fields, but two of the three projections involved are not divergence-free for arbitrary vector fields, and so do not permit the definition of, for example, the poloidal part of a field. This is evident from (3.5) and (3.6).

Here the mean $\mathscr{M}$ and quasipoloidal $\mathscr{Q}$ subspaces are partitioned with the result that the space of d.p. vector fields can be decomposed into three mutually orthogonal divergence-free subspaces (those of Schmitt and Wahl), and two subspaces orthogonal to each other and to each of the divergence-free subspaces. It is unnecessary to partition $\mathscr{T}$, since by (3.7), all its elements are divergence-free. To decompose $\mathscr{M}$ and $\mathscr{Q}$, we find the condition characterising divergence-free elements, and then the condition characterising elements orthogonal to all such. Thus

$$
\begin{array}{rlrl}
\mathscr{B} & =\{\boldsymbol{b}: \boldsymbol{b} \in \mathscr{M}, \nabla \cdot \boldsymbol{b}=0\}, & \mathscr{N} & =\mathscr{M}-\mathscr{B}, \\
\mathscr{P} & =\{\boldsymbol{p}: \boldsymbol{p} \in \mathscr{Q}, \nabla \cdot \boldsymbol{p}=0\}, & \mathscr{S}=\mathscr{Q}-\mathscr{P} .
\end{array}
$$


TABLE 2. Quinquepartite decomposition of d.p. vector fields.

\begin{tabular}{|c|c|c|}
\hline Space & Projection & Scalar representation \\
\hline $\mathscr{B}$ & $\mathbf{P}_{\mathscr{B}} \boldsymbol{v} \equiv\left\langle v_{x}\right\rangle \mathbf{i}_{x}+\left\langle v_{y}\right\rangle_{\square} \mathbf{i}_{y}+\left\langle v_{z}\right\rangle_{\square} \mathbf{i}_{z}$ & $\begin{array}{l}b=U i_{x}+V i_{y}+W i_{z} \\
\nabla U=\nabla_{\square} V=\nabla_{\square} W=0\end{array}$ \\
\hline $\mathscr{N}$ & $\mathbf{P}_{\mathscr{N}} \boldsymbol{v} \equiv\left(\left\langle v_{x}\right\rangle_{\square}-\left\langle v_{x}\right\rangle\right) \mathbf{i}_{x}$ & $\begin{array}{l}n=N \mathbf{i}_{x} \\
\nabla_{\square} N=\mathbf{0},(N)=0\end{array}$ \\
\hline $\mathscr{P}$ & $\begin{array}{l}\mathrm{P}_{\mathscr{g}} v \equiv-\nabla_{\square}^{2} \Psi \mathrm{i}_{x}+\nabla_{\square} \mathrm{D} \Psi, \text { where } \\
\nabla^{2} \Psi=-\left(v_{x}-\left\langle v_{x}\right\rangle_{\square}\right)+\mathrm{D} \nabla_{\square}^{-2} \nabla_{\square} \cdot v \\
\langle\Psi\rangle_{\square}=0 \\
\mathrm{D} \Psi=\nabla_{\square}^{-2} \nabla_{\square} \cdot v \quad\left(x=x_{0}, x_{1}\right)\end{array}$ & $\begin{array}{l}p=-\nabla_{a}^{2} \Psi i_{x}+\nabla_{\square} \mathrm{D} \Psi \\
\langle\Psi\rangle_{\square}=0\end{array}$ \\
\hline $\mathscr{S}$ & $\begin{array}{l}P_{\mathcal{S}} v \equiv-\nabla \sigma, \text { where } \\
\nabla^{2} \sigma=-\left(\nabla \cdot v-\langle\nabla \cdot v\rangle_{\square}\right) \\
\langle\sigma\rangle_{\square}=0 \\
\sigma=0 \quad\left(x=x_{0}, x_{1}\right)\end{array}$ & $\begin{array}{l}s=-\nabla \sigma \\
\langle\sigma\rangle_{\square}=0 \\
\sigma=0 \quad\left(x=x_{0}, x_{1}\right)\end{array}$ \\
\hline $\mathscr{T}$ & $\begin{array}{l}P_{\mathscr{g}} v \equiv-\Lambda \tau, \text { where } \\
\tau=-\nabla_{\square}^{-2} \Lambda \cdot v\end{array}$ & $\begin{array}{l}t=-\Lambda \tau \\
\langle\tau\rangle_{\square}=0\end{array}$ \\
\hline
\end{tabular}

The subspace $\mathscr{S}$ is called scaloidal by analogy with usage for the spherical case $[6,1]$. The subspace $\mathscr{N}$ contains only no-mean normal fields.

The resulting five subspaces are listed in Table 2 . In each case, the projection given in Table 2 is the only one compatible with the given definition of its space. In particular, the boundary conditions on the Poisson equations for the poloidal and scaloidal projections had to be so chosen to ensure that the projection was indeed idempotent and Hermitian, and that all divergence-free quasipoloidal fields were poloidal.

The table also lists scalar representations of general members of each space; in each case, definition of the space as the collection of all possible fields having the given representation is equivalent to the original definition; further, the scalars are unique.

General solutions of the space Poisson equations in the poloidal and scaloidal projections were given in Section 3.1.2. Note that if $\nabla \cdot v=0$, the space Poisson equation for the poloidal projection reduces to

$$
\nabla^{2} \Psi=-\nabla^{2} \nabla_{\square}^{-2}\left(v_{x}-\left\langle v_{x}\right\rangle_{\square}\right), \quad \mathrm{D} \Psi=-\mathrm{D} \nabla_{\square}^{-2}\left(v_{x}-\left\langle v_{x}\right\rangle_{\square}\right), \quad\left(x=x_{0}, x_{1}\right),
$$

so that $\Psi=-\nabla_{\square}^{-2}\left(v_{x}-\left\langle v_{x}\right\rangle_{\square}\right)$ and the projection reduces again to that of Schmitt and Wahl [23, Eq. 7], as in Section 3.2.1.

4.1. Proof for the scaloidal subspace The methods used to prove the statements in Table 2 are similar for each space, so only the proof for scaloidal space is given here.

Proof. We prove first that the projection $\mathrm{P}_{\mathscr{S}}$ is Hermitian and then second that $\mathscr{S}^{\prime}$ 
defined by (4.1), $\mathscr{S}^{\prime \prime}$ defined as the class of fields with the given scalar representation, and $\mathscr{S}$ defined by the given projection are equivalent, that is, $\mathscr{S}^{\prime} \subseteq \mathscr{S}^{\prime \prime} \subseteq \mathscr{S} \subseteq \mathscr{S}^{\prime}$. That $\mathrm{P}_{\mathscr{S}}^{2}=\mathrm{P}_{\mathscr{S}}$ follows from the second part of the proof.

First, $\mathbf{P}_{\mathscr{S}}$ is Hermitian, since if $\sigma$ and $\tau$ are the scaloidal scalars for arbitrary d.p. fields $\mathbf{v}$ and $\mathbf{w},\left\langle\mathbf{P}_{\mathscr{S}} \mathbf{v}, \mathbf{w}\right\rangle=-\langle\nabla \sigma, \mathbf{w}\rangle=\langle\nabla \sigma, \nabla \tau\rangle=\left\langle\mathrm{P}_{\mathscr{S}} \mathbf{v}, \mathrm{P}_{\mathscr{S}} \mathbf{w}\right\rangle$. We have twice used the condition that $\sigma$ vanishes at the ends $x=x_{0}, x_{1}$.

Second,

(1) $v \in \mathscr{S}^{\prime} \subseteq \mathscr{Q}$ implies $v=-r \mathbf{i}_{x}-\nabla_{\square} s$ where $\langle r\rangle_{\square}=\langle s\rangle_{\square}=0$. To prove $v \in \mathscr{S}^{\prime \prime}$, we use the fact that $v \in \mathscr{S}^{\prime} \perp \mathscr{P}$ implies $\mathrm{P}_{\mathscr{P}} v=0$. In particular, $\mathbf{i}_{x} \cdot \mathrm{P}_{\mathscr{P}} v=-\nabla_{\square}^{2} \Psi=0$. Therefore, by the uniqueness result of Section 2.1.6, $\Psi=0$ where

$$
\begin{aligned}
\nabla^{2} \Psi & =-\left(v_{x}-\left\langle v_{x}\right\rangle_{\square}\right)+\mathrm{D} \nabla_{\square}^{-2} \nabla_{\square} \cdot v \\
& =-\left(-r-\langle-r\rangle_{\square}\right)+\mathrm{D} \nabla_{\square}^{-2} \nabla_{\square} \cdot\left(-\nabla_{\square} s\right)=-r-\mathrm{D} s
\end{aligned}
$$

and at $x=x_{0}$ and $x_{1}, \mathrm{D} \Psi=\nabla_{\square}^{-2} \nabla_{\square} \cdot\left(-\nabla_{\square} s\right)=-s$. Thus $s\left(x_{0}\right)=s\left(x_{1}\right)=0$ and $r=\mathrm{D} s$, that is, $v=-\mathrm{D} s-\nabla_{\square} s=-\nabla s$. Therefore $\mathscr{S}^{\prime} \in \mathscr{S}^{\prime \prime}$.

(2) For the typical element of $\mathscr{S}^{\prime \prime}, \mathrm{P}_{\mathscr{S}}(-\nabla \sigma)=-\nabla s$ where

$$
\begin{aligned}
\nabla^{2} s & =-\left(-\nabla \cdot \nabla \sigma+\langle\nabla \cdot \nabla \sigma\rangle_{\square}\right) \\
& =\nabla^{2} \sigma-\left\langle\mathrm{D}^{2} \sigma\right\rangle_{\square}-\left\langle\nabla_{\square}^{2} \sigma\right\rangle_{\square}=\nabla^{2} \sigma-0-0=\nabla^{2} \sigma,
\end{aligned}
$$

$\langle s\rangle_{\square}=0=\langle\sigma\rangle_{\square}$ and $s\left(x_{0}\right)=s\left(x_{1}\right)=0=\sigma\left(x_{0}\right)=\sigma\left(x_{1}\right)$. Therefore, by the uniqueness result of Section 3.1.2,s $=\sigma$ and $\mathrm{P}_{\mathscr{S}}(-\nabla \sigma)=-\nabla s=-\nabla \sigma$. Thus $\mathscr{S}^{\prime \prime} \subseteq \mathscr{S}$. (This also proves that $\mathrm{P}_{\mathscr{S}}$ is idempotent.)

(3) For arbitrary d.p. $v, \mathrm{P}_{\mathscr{S}} v=-\nabla \sigma$ where $\langle\sigma\rangle_{\square}=0$ and $\sigma\left(x_{0}\right)=\sigma\left(x_{1}\right)=0$. Then

$$
\mathrm{P}_{\mathscr{Q}} \mathrm{P}_{\mathscr{S}} v=-\mathrm{D} \sigma \mathbf{i}_{x}-\nabla_{\square} \sigma=-\nabla \sigma=\mathrm{P}_{\mathscr{S}} v
$$

so $\mathscr{S} \subseteq \mathscr{Q}$. Also $\mathrm{P}_{\mathscr{P}} \mathrm{P}_{\mathscr{S}} v=-\nabla_{\square}^{2} \Psi \mathbf{i}_{x}+\nabla_{\square} \mathrm{D} \Psi$ where

$$
\nabla^{2} \Psi=-\mathrm{D} \sigma+\mathrm{D} \nabla_{\square}^{-2} \nabla_{\square} \cdot\left(-\nabla_{\square} \sigma\right)=0,
$$

$\langle\Psi\rangle_{\square}=0$, and at $x=x_{0}$ and $x=x_{1}$,

$$
\mathrm{D} \psi=\nabla_{\square}^{-2} \nabla_{\square} \cdot\left(-\nabla_{\square} \sigma\right)=-\sigma=0 .
$$

Therefore, by the uniqueness result of Section 3.1.2, $\Psi=0$ and $\mathrm{P}_{\mathscr{P}} \mathrm{P}_{\mathscr{S}}=0$, that is, $\mathscr{S} \perp \mathscr{P}$ and $\mathscr{S} \subseteq \mathscr{S}^{\prime}$.

The scalar representation (Table 2) of a scaloidal field is unique. Say $v \in \mathscr{S}=$ $-\nabla \sigma=-\nabla s$ and $\langle\sigma\rangle_{\square}=\langle s\rangle_{\square}=0$. Then $\nabla(s-\sigma)=0$ so that $s-\sigma=\langle s-\sigma\rangle_{\square}=$ $\langle s\rangle_{\square}-\langle\sigma\rangle_{\square}=0$, that is, $s=\sigma$. 
4.2. Quinquepartite Fourier decomposition The Fourier projections

$$
\mathrm{P}_{\ell} v \equiv\left\langle e_{\ell}, v_{x}\right\rangle_{\square} e_{\ell} \mathbf{i}_{x}+\left\langle e_{\ell}, v_{\eta_{\ell}}\right\rangle_{\square} e_{\ell} \mathbf{i}_{\eta_{\ell}}+\left\langle e_{\ell}, v_{\xi_{\ell}}\right\rangle_{\square} e_{\ell} \mathbf{i}_{\xi_{\ell}}
$$

commute with each of $\mathrm{P}_{\mathscr{N}}, \mathrm{P}_{\mathscr{B}}, \mathrm{P}_{\mathscr{S}}, \mathrm{P}_{\mathscr{P}}$ and $\mathrm{P}_{\mathscr{T}}$. For $l_{y}=l_{z}=0$

$$
\mathrm{P}_{0} \mathrm{P}_{\mathscr{N}}=\mathrm{P}_{\mathscr{N}} \mathrm{P}_{0}=\mathrm{P}_{\mathscr{N}} \quad \text { and } \quad \mathrm{P}_{0} \mathrm{P}_{\mathscr{B}}=\mathrm{P}_{\mathscr{B}} \mathrm{P}_{0}=\mathrm{P}_{\mathscr{B}}
$$

while $\mathrm{P}_{0}$ is orthogonal to each of $\mathrm{P}_{\mathscr{S}}, \mathrm{P}_{\mathscr{P}}$ and $\mathrm{P}_{\mathscr{T}}$ (indeed $\mathrm{P}_{0}=\mathrm{P}_{\mathscr{A}}$ ). For nonzero $\ell$, $\mathrm{P}_{\ell}$ is orthogonal to $\mathrm{P}_{\mathscr{N}}$ and $\mathrm{P}_{\mathscr{B}}$ while

$$
\begin{aligned}
\mathrm{P}_{\mathscr{S}_{\ell}} v & \equiv \mathrm{P}_{\ell} \mathrm{P}_{\mathscr{S}} v=\mathrm{P}_{\mathscr{S}} \mathrm{P}_{\ell} v=-\mathrm{D} \tilde{\sigma}_{\ell} e_{\ell} \mathbf{i}_{x}-\sqrt{-1} \kappa_{\ell} \tilde{\sigma}_{\ell} e_{\ell} \mathbf{i}_{\eta_{\ell}}, \\
\mathrm{P}_{\mathscr{P}} v & \equiv \mathrm{P}_{\ell} \mathrm{P}_{\mathscr{P}} v=\mathrm{P}_{\mathscr{P}} \mathrm{P}_{\ell} v=\kappa_{\ell}^{2} \widetilde{\Psi}_{\ell} e_{\ell} \mathbf{i}_{x}+\sqrt{-1} \kappa_{\ell} \mathrm{D} \widetilde{\Psi}_{\ell} e_{\ell} i_{\eta_{\ell}} \quad \text { and } \\
\mathrm{P}_{\mathscr{T}_{\ell}} v & \equiv \mathrm{P}_{\ell} \mathrm{P}_{\mathscr{T}} v=\mathrm{P}_{\mathscr{T}} \mathrm{P}_{\ell} v=-\sqrt{-1} \kappa_{\ell} \tilde{\tau}_{\ell} e_{\ell} \mathbf{i}_{\zeta_{\ell}} .
\end{aligned}
$$

Thus each Fourier-scaloidal (4.2) and Fourier-poloidal (4.3) component field is parallel, and each Fourier-toroidal component field (4.4) normal, to the $x \eta_{\ell}$-plane. All three types of Fourier component field are two dimensional, being independent of $\zeta_{\ell}$ and $\eta_{\ell}$-periodic, depending on $\eta_{\ell}$ only via $e_{\ell}(2.10)$. A Fourier-poloidal component can also be written as

$$
\mathbf{P}_{\mathscr{P}_{\ell}} v=\left(-\mathbf{i}_{x} \frac{\partial}{\partial \eta_{\ell}}+\mathbf{i}_{\eta_{\ell}} \mathrm{D}\right)\left(\sqrt{-1} \kappa_{\ell} \widetilde{\Psi}_{\ell} e_{\ell}\right),
$$

so that the expression in the second pair of parentheses is a stream-function for $\mathrm{P}_{\mathscr{G}} v$ in the $x \eta_{\ell}$-plane.

\section{Discussion and conclusions}

5.1. Example: divergence-free field vanishing on $|x|=1$ As an example of the quinquepartite decomposition of a d.p. divergence-free vector field, consider a velocity field for an incompressible fluid between the walls $x=-1$ and $x=1$. The field $v$ vanishes on the walls.

This implies that the poloidal scalar for the field vanishes along with its $x$-derivative at the walls. The monomials $\left\{x^{n}\right\}_{n=0}^{\infty}$ form a basis for functions of $x$ on $(-1,1)$, and $\left\{\left(1-x^{2}\right)^{2} x^{n}\right\}_{n=0}^{\infty}$ for the subspace of functions vanishing along with their derivatives at the end points. Recalling [7] that the ultraspherical polynomials $C_{n}^{(\alpha)}$ satisfy

$$
\int_{-1}^{1}\left(1-x^{2}\right)^{\alpha-1 / 2} C_{n}^{(\alpha)}(x) C_{m}^{(\alpha)}(x) \mathrm{d} x= \begin{cases}0, & n \neq m \\ h_{n}^{(\alpha)}, & n=m,\end{cases}
$$


where

$$
h_{n}^{(\alpha)}=\frac{2^{1-2 \alpha} \pi \Gamma(n+2 \alpha)}{n !(n+\alpha)\{\Gamma(\alpha)\}^{2}},
$$

we find that an orthonormal basis for this subspace is

$$
p_{n}(x) \equiv \frac{\left(1-x^{2}\right)^{2}}{\sqrt{h_{n}^{(9 / 2)}}} C_{n}^{(9 / 2)}(x) .
$$

Stream-lines of real parts of a few poloidal fields of the form $(\nabla \times)^{2}\left(p_{n} e_{\ell} \mathbf{i}_{x}\right)$ with small $n$ and $\ell$ are illustrated in Figure 3.

For the toroidal fields. the end condition $v( \pm 1)=0$ implies only $\tau( \pm 1)=0$, so that an orthonormal basis again consists of weighted ultraspherical polynomials but with $\alpha=5 / 2$ :

$$
t_{n}(x) \equiv \frac{1-x^{2}}{\sqrt{h_{n}^{(5 / 2)}}} C_{n}^{(5 / 2)}(x)
$$

(see, for example, [19, Eq. 44], noting that $C_{n}^{(\alpha)} \equiv P_{n}^{(\alpha-1 / 2, \alpha-1 / 2)}$, where $P_{n}^{(\alpha-1 / 2, \alpha-1 / 2)}$ are Jacobi polynomials). The stream-lines are simply lines of constant $x$ and $\eta_{\ell}$, so some toroidal fields are illustrated in Figure 4 by velocity contours in planes of constant $\zeta_{l}$.

The vector modes of this section are equivalent to some used previously [19]; however, being expressed in regular Cartesian coordinates has obscured their simple geometrical properties, namely the two-dimensionality of the poloidal modes and the unidirectionality of the toroidal modes. This is just as the special threedimensional significance of two-dimensional solutions of the Orr-Sommerfeld equation [5, page 155] only becomes evident after Squire's transformation [26].

5.2. Further properties of the decomposition Figure 4 bears a certain resemblance to the first row of Figure 3. This is not an accident. It is has been proven elsewhere [12] that if a three-dimensional velocity field has zero gradient in some direction then the component of velocity in that direction is constant along vortex-lines (curves parallel to the curl of the velocity). And, indeed, the curl of any toroidal field is poloidal.

THEOREM 5.1. $\nabla \times \mathscr{T}=\mathscr{P}$.

Proof. The curl of a typical toroidal element (Table 2) is

$$
\nabla \times(-\Lambda \tau)=-\nabla_{\square}^{2} \tau \mathbf{i}_{x}+\nabla_{\square} \mathrm{D} \tau
$$

which is the most general form of a poloidal field (Table 2). 

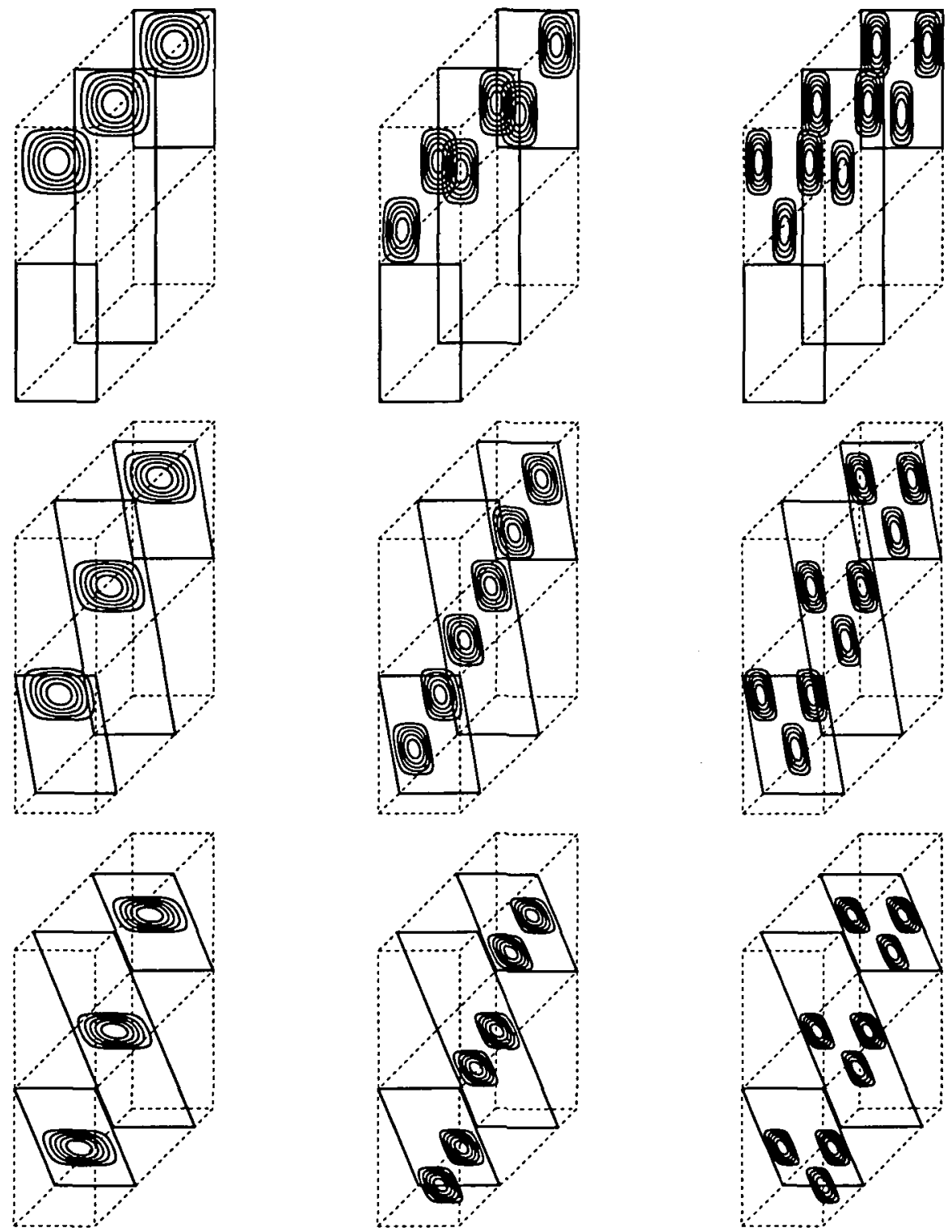

FIGURE 3. Stream-lines of poloidal fields with stream-functions $-\kappa_{\ell} p_{n}(x) \sin \kappa_{\ell} \eta_{l}$. Here $l_{z}=0,1,2$ by row and $n=0,1,2$ by column; $l_{y}=1$ in all; $k$ and $\gamma$ as in Figures 1 and 2 . Only stream-lines corresponding to 'positive' rolls of a single period cell are shown; counterrotating rolls are symmetrically disposed. Stream-lines lie in the planes $\zeta_{l}=0, \pi \cos \beta_{l} / k \sin \gamma, 2 \pi \cos \beta_{l} / k \sin \gamma$, as marked by solid lines. Dashes mark boundaries of two $y z$-period cells and $|x|=1$. 

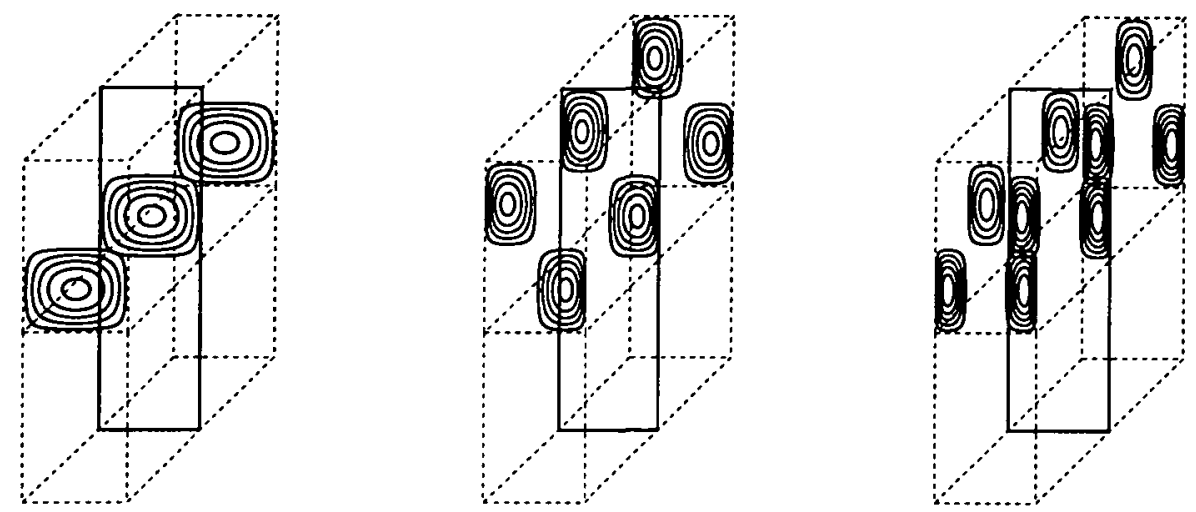

FIGURE 4. Contours of constant velocity of three toroidal fields. $n=0,1,2 ; l_{y}=1$ and $l_{z}=0$ in all; projection and periodicity ( $k$ and $\gamma$ ) as in Figures 1-3.

For the other subspaces the corresponding results are:

$$
\begin{aligned}
& \nabla \times \mathrm{P}_{\mathscr{P}} \boldsymbol{v}=-\boldsymbol{\Lambda} \nabla^{2} \Psi \in \mathscr{T}, \\
& \nabla \times \mathrm{P}_{\mathscr{B}} \boldsymbol{v}=-\mathrm{D}\left\langle v_{z}\right\rangle_{\square} \mathbf{i}_{y}+\mathrm{D}\left\langle v_{y}\right\rangle_{\square} \mathbf{i}_{z} \in \mathscr{B}, \\
& \nabla \times \mathrm{P}_{\mathscr{S}} \boldsymbol{v}=0 \text { and } \boldsymbol{\nabla} \times \mathrm{P}_{\mathscr{N}} \boldsymbol{v}=0 .
\end{aligned}
$$

Theorem 5.1 and (5.1) are analogous to known results for spherical poloidal and toroidal fields [3, page 623]. That the divergence-free subspaces $\mathscr{S}$ and $\mathscr{N}$ are irrotational shows the relation to the Helmholtz decomposition; note, however, that there are nonzero irrotational members of $\mathscr{P}$ and $\mathscr{B}$, for example,

$$
\boldsymbol{p}=(\nabla \times)^{2}\left\{\mathbf{i}_{x} \cosh (k x \cos \gamma) \cos (k y \cos \gamma)\right\}
$$

is an irrotational poloidal field, and any uniform vector field is an irrotational member of $\mathscr{B}$.

5.3. Conclusion The result of the analysis is a decomposition of the space of arbitrary three-dimensional d.p. vector fields into five subspaces: three divergencefree, coinciding with those of Schmitt and Wahl [23], and two orthogonal to these and each other.

Further, one each of these groups of three and two contains only elements constant in the two periodic directions; these two are the 'mean' subspaces. Of the three nonmean subspaces, the Fourier decomposition reduces two to the sum of subspaces containing only two-dimensional vector fields (that is, having two nonzero components and independent of the third coordinate in an appropriately rotated Cartesian system) and the other to the sum of subspaces containing only divergence-free unidirectional 
fields. This reduction of a three-dimensional field to a series of two-dimensional fields should simplify the analysis and solution of three-dimensional vector field problems.

Each of the five subspaces are characterised both by projections and by scalar representations. In the solution of problems, these should be useful for the reduction of known and unknown vector fields, respectively.

\section{Acknowledgements}

The author thanks Assoc. Prof. S. W. Armfield for his helpful insights and suggestions. The free computer algebra systems GNU Calc and GNU Maxima were useful in checking some of the results.

\section{References}

[1] G. Backus, "Poloidal and toroidal fields in geomagnetic field modelling", Rev. Geophysics 24 (1) (1986) 75-109.

[2] F. H. Busse, "On Howard's upper bound for heat transport by turbulent convection", J. Fluid Mech. 37 (1969) 457-477.

[3] S. Chandrasekhar, Hydrodynamic and Hydromagnetic Stability (Dover, New York, 1981).

[4] R. M. Clever and F. H. Busse, "Tertiary and quaternary solutions for plane Couette flow", J. Fluid Mech. 344 (1997) 137-153.

[5] P. G. Drazin and W. H. Reid, Hydrodynamic Stability, 2nd ed. (Cambridge University Press, Cambridge, 2004).

[6] W. M. Elsasser, "Induction effects in terrestrial magnetism. I. Theory", Phys. Rev. 69 (1946) 106-116.

[7] U. W. Hochstrasser, "Orthogonal polynomials", in Handbook of Mathematical Functions (Eds. M. Abramowitz and I. Stegun), (Dover, New York, 1965) Ch. 22, 771-802.

[8] C. A. Holt, Introduction to Electromagnetic Fields and Waves (Wiley, New York, 1963).

[9] D. D. Joseph, Stability of Fluid Motions I (Springer, Berlin, 1976).

[10] H. Lamb, Hydrodynamics, 6th ed. (Cambridge University Press, Cambridge, 1932).

[11] G. D. Mallinson and G. de Vahl Davis, "The method of the false transient for the solution of coupled elliptic equations", J. Comput. Phys. 12 (1973) 435-461.

[12] G. D. McBain, "Fully developed laminar buoyant flow in vertical cavities and ducts of bounded section", J. Fluid Mech. 401 (1999) 365-377.

[13] G. D. McBain, "Convection in a horizontally heated sphere", J. Fluid Mech. 438 (2001) 1-10.

[14] G. D. McBain and S. W. Armfield, "Natural convection in a vertical slot: Accurate solution of the linear stability equations", ANZIAM J. 45(E) (2004) C92-C105.

[15] V. Mityushev and P. M. Adler, "Longitudinal permeability of spatially periodic rectangular arrays of circular cylinders I. A single cylinder in the unit cell", Z Angew. Math. Mech. 82 (5) (2002) 335-345.

[16] H. K. Moffatt, Magnetic Field Generation in Electrically Conducting Fluids (Cambridge University Press, Cambridge, 1978).

[17] M. Nagata, "Bifurcations in Couette flow between almost corotating cylinders", J. Fluid Mech. 169 (1986) 229-250. 
[18] A. E. Perry and M. S. Chong, "A description of eddying motions and flow patterns using critical point concepts", Ann. Rev. Fluid Mech. 19 (1987) 125-155.

[19] A. Rauh, S. Page and A. Stille, "Energy inconsistency of Galerkin approximations for plane Couette flow”, Rep. Math. Phys. 54 (2004) 23-40.

[20] Rayleigh, Lord, "On convection currents in a horizontal layer of fluid, when the higher temperature is on the underside", Lond., Edin. \& Dubl. Phil. Mag. \& J. Sci. 32 (192) (1916) 529-546.

[21] O. Reynolds, "On the theory of lubrication and its application to Mr. Beauchamp Tower's experiments, including an experimental determination of the viscosity of olive oil", Phil. Trans. R. Soc. Lond 177 (1886) 157-234.

[22] D. W. Ruth, "On the transition to transverse rolls in an infinite vertical fluid layer-a power series solution", Intl J. Heat Mass Transfer 22 (1979) 1199-1208.

[23] B. J. Schmitt and W. von Wahl, "Decomposition of solenoidal fields into poloidal fields, toroidal fields and the mean flow. Applications to the Boussinesq-equations", Lecture Notes in Math. 1530 (1992) 291-305.

[24] L. A. Segel, "The non-linear interaction of a finite number of disturbances to a layer of fluid heated from below", J. Fluid Mech. 21 (1965) 359-384.

[25] M. Sherman, "Toroidal and poloidal field representation for convective flow within a sphere", Phys. Fluids 11 (1968) 1895-1900.

[26] H. B. Squire, "On the stability for three-dimensional disturbances of viscous fluid between parallel walls", Proc. R. Soc. Lond., Ser. A 142 (841) (1933) 621-628.

[27] J. von Neumann, Mathematical Foundations of Quantum Mechanics, Trans. R. T. Beyer (Princeton University Press, Princeton, New Jersey, 1955).

[28] L. Waldmann, "Zur Theorie des Gastrennungsverfahrens von Clusius und Dickel", Die Naturwissenschafien 27 (14) (1939) 230-231. 